\title{
Correction to: Analysis of IKBKG/NEMO gene in five Japanese cases of incontinentia pigmenti with retinopathy: fine genomic assay of a rare male case with mosaicism
}

\author{
Muhammad Nazmul Haque - Masafumi Ohtsubo (D) Sachiko Nishina - Shiro Nakao Kazue Yoshida • \\ Katsuhiro Hosono - Kentaro Kurata - Kentaro Ohishi • Maki Fukami (D) Miho Sato • Yoshihiro Hotta (D) \\ Noriyuki Azuma · Shinsei Minoshima
}

Published online: 27 January 2021

(c) The Author(s), under exclusive licence to The Japan Society of Human Genetics 2021

Correction to: Journal of Human Genetics https://doi.org/10.1038/s10038-020-00836-3

The authors of the above paper regret to inform a mistake in the original version. The number of the analyzed parents were not correct in the last paragraph of the "Introduction" and in the first paragraph of "Patients and their clinical manifestations" part. In both places, the authors described "patients together with parents of two of them"; but actually, it must be "patients together with parents of three of them". This correction does not affect all of the remaining parts of this paper including the data interpretaion and conclusion. The authors would like to apologize for this mistake. 